\title{
Influence of material property variability on the thickness in sheet metal subjected to the hydraulic bulging
}

\author{
Lucian LĂZĂRESCU ${ }^{1, a}$, Dorel BANABIC ${ }^{1, b}$ \\ ${ }^{1}$ CERTETA Research Centre, Technical University of Cluj-Napoca, Cluj-Napoca, Romania \\ alucian.lazarescu@tcm.utcluj.ro, b banabic@tcm.utcluj.ro
}

Keywords: Sheet metal forming, Hydraulic bulge test, Sensitivity analysis, AutoForm-Sigma.

\begin{abstract}
In the sheet metal forming processes, the inherent unavoidable noise in material property, which can vary inside and between batches as well as between suppliers, constitutes an important source of uncertainty which could result in rejection of some formed parts. In the process design phase, a successful tool for the sensitivity analysis proved to be the finite element codes, in which case the material parameters are used as input into the yield criterion. This paper investigates the thickness sensitivity due to the noise in material property using the finite element simulation of hydraulic bulge test. The BBC 2005 yield criterion with eight constitutive parameters, which is implemented in the commercially finite element code AutoForm has been used in this study.
\end{abstract}

\section{Introduction}

The mechanical property variability of sheet metals used in the forming processes is an inherent property which can lead to high rate of scraps. Due to the noise in the material property, a single finite element simulation of a sheet forming process is insufficient to guarantee the robustness of that process. Therefore in the design phase of a forming process in order to verify the robustness it is required to consider the effect of the material property variability.

The noise in material property, as for example yield stress, yield strength and anisotropy coefficients, may vary between batches and between suppliers. According to the standard specification EN 10130: 2006, the DC04 steel has a tensile strength value between 270 and 350 $\mathrm{MPa}$. The variability of mechanical property of sheet metal and their effect in a forming process has been studied by other researcher. Karthik et al. [1] have carried out tensile and formability tests independently at three laboratories using more than 45 coils of materials. They concluded that coilto-coil variations are higher than the observed test-to-test. Paraianu et al. [2] have statistically investigated the variability of mechanical parameters of a DC04 steel sheet. They revealed that the anisotropy coefficients have the most important variation. Banabic and Vos [3] and Paraianu et al. [4] found that the material parameters $\sigma_{0}, \sigma_{90}, \sigma_{\mathrm{b}}$ and $\mathrm{n}$, have a height influence on the forming limit curve. Many authors investigated the effect of material property variability on springback $[5,6]$. Sugar et al. [7] have determined the significant parameters influencing the thinning of spun parts by ANOVA method. Gödel et al [8] investigated the effect of material model and material property variability on the results within the stochastic simulation. Atzema et al. [9] investigated the effect of material models used in simulation on the prediction of process variations and scrap rate. They concluded that the effect of models on the variation itself is small, while the effect on the scrap rate is significant. In the context of robust process design by taking into account the material parameters variability there are several finite element simulation codes on the market such AutoForm Sigma and PAM-STAMP whose merits were discussed in [9]. Studies, using AutoForm Sigma code, of the effect of material property variability on thinning have been presented by Banabic [10].

In this paper the effect of material property variability on the thickness is investigated using the finite element simulation of sheet metals in the hydraulic bulge test. A finite element model of hydraulic bulging has been developed using the AutoForm code and validated by comparison the predicted results with the experimental data provided by the ARAMIS system. Four zones within the specimen and six material parameters from the eight parameters BBC 2005 material model as noise inputs were considered in the paper. 


\section{Investigated material}

In this paper a DC04 cold rolled low carbon steel sheet with the nominal thickness of $0.85 \mathrm{~mm}$ has been considered, whose mechanical property are given in Table 1 . The yield stresses $\left(\sigma_{0}, \sigma_{45}, \sigma_{90}\right)$ and the anisotropy coefficients $\left(\mathrm{r}_{0}, \mathrm{r}_{45}, \mathrm{r}_{90}\right)$ have been obtained by tensile tests on the specimen cut at different orientations defined by $0^{\circ}, 45^{\circ}$, and $90^{\circ}$ degrees with respect to the sheet rolling direction (RD). To do this a tensile test machine Zwick Roell Z150 has been used, which is equipped with an extensometer for the measurement and acquiring the strains in two directions. The biaxial yield stress $\left(\sigma_{b}\right)$ has been determined by hydraulic bulge test using the equivalent plastic work principle, as is described in the paper [11].

Table 1 Mechanical property of the DC04 sheet steel (thickness: $0.85 \mathrm{~mm}$ )

\begin{tabular}{|c|c|c|c|c|c|c|c|}
\hline \multicolumn{8}{|c|}{ Material parameters } \\
\hline$\sigma_{0}[\mathrm{MPa}]$ & $\sigma_{45}[\mathrm{MPa}]$ & $\sigma_{90}[\mathrm{MPa}]$ & $\sigma_{b}[\mathrm{MPa}]$ & $\mathrm{r}_{0}$ & $\mathrm{r}_{45}$ & $\mathrm{r}_{90}$ & $\mathrm{r}_{\mathrm{b}}$ \\
\hline 196 & 211 & 206 & 227 & 1.96 & 1.3 & 2.19 & 0.957 \\
\hline \multicolumn{8}{|c|}{ Hardening law (Swift) coefficients } \\
\hline $\mathrm{C}[\mathrm{MPa}]$ & $\mathrm{n}[-]$ & $\varepsilon_{0}[-]$ & & & & & \\
\hline 547.16 & 0.238 & 0.01 & & & & & \\
\hline
\end{tabular}

The eight parameters BBC 2005 yield criterion has been used to model the yield locus for the DC04 steel (Fig.1a). The experimental material parameters given in Table 1 have been used as input data in the identification procedure to obtain the coefficients of the yield criterion.

From literature is a known that the Swift's law is a good approximation of the true stress-strain curve for steels, which show a continuous work-hardening behaviour (Fig. 1b). The parameters of Swift equation (Eq. (1)) have been obtained by the least-square fitting of five stress-strain experimental curves obtained by tensile tests on specimens cut at $0^{\circ}$ angle with respect to the rolling direction of sheet. The obtained coefficients are given in Table 1.

$$
\sigma=C\left(\varepsilon_{0}+\varepsilon_{p}\right)^{n}
$$

where, $\sigma$ is the flow stress; $\mathrm{n}$ is the strain hardening exponent; $\varepsilon_{0}$ is the initial plastic strain and $\varepsilon_{\mathrm{p}}$ is the plastic strain.

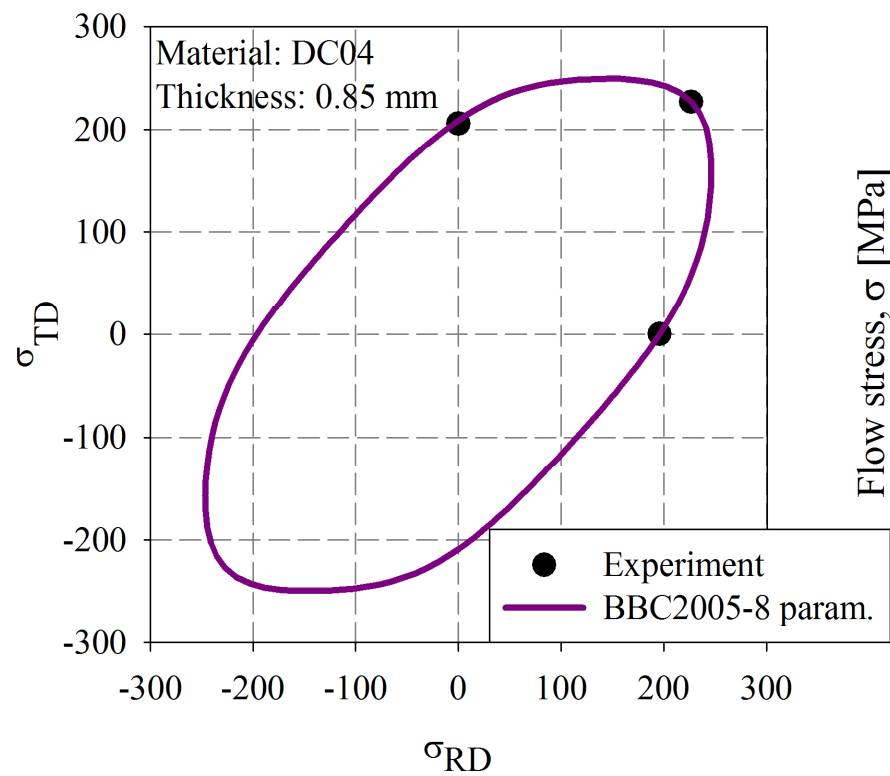

a)

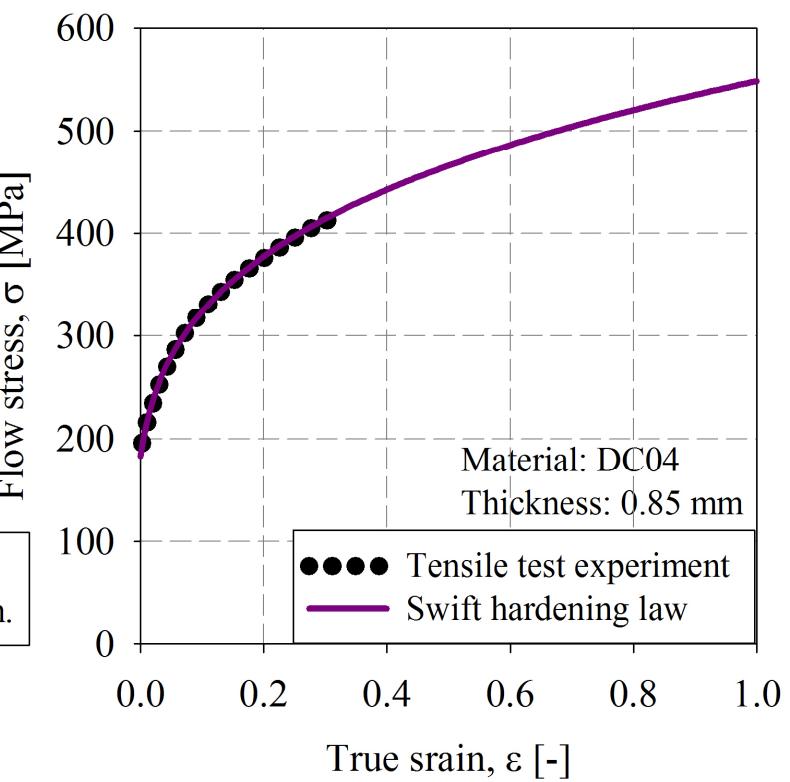

b)

Fig. 1. The yield locus described by the eight parameters BBC 2005 yield criterion (a) and the associated strain hardening curve by Swift (b) 


\section{Experiments}

In order to investigate the influence of the DC04 material property variability on the thickness, the geometry of specimen from the hydraulic bulge test with circular die aperture has been used. It is known that in the polar zone of the bulged specimen the material is in a biaxial tensile stress state, while in the area near the fillet radius of die, the material is in a uniaxial tensile stress state. Additionally, by choosing different zones within the specimen at $0^{\circ}, 45^{\circ}$, and $90^{\circ}$ angles with respect to the rolling direction it is expected to occur a different dominant material parameter which influences the specimen thickness. One may conclude that this test is characterized by the existence of two states of the stresses (biaxial and uniaxial tensile stress states) which is significant for serial production of some parts, as for example deep drawing and sheet hydroforming.

Experimental setup. The hydraulic bulging experiment is carried out using a universal sheet testing machine of the type Erichsen, model 142-20 and an optical ARAMIS 4M system [12] for the strain measurement, as shown in Fig. 2. A die with aperture of $100 \mathrm{~mm}$ and the fillet radius $6 \mathrm{~mm}$ has been used in the experiment. In the hydraulic bulge test, the specimen is firmly clamped on its periphery between a die and a blank holder. Then, the specimen is deformed trough the circular aperture of the die under the action of increasing pressure applied on its bottom surface. The hydraulic pressure and the images of deformed specimen from the beginning to the end of the test are recorded by the ARAMIS system. Using the ARAMIS software, the geometry of specimen is reconstituted and the strains are computed. Fig. 3 shows an example of the specimen geometry and strains distributions.
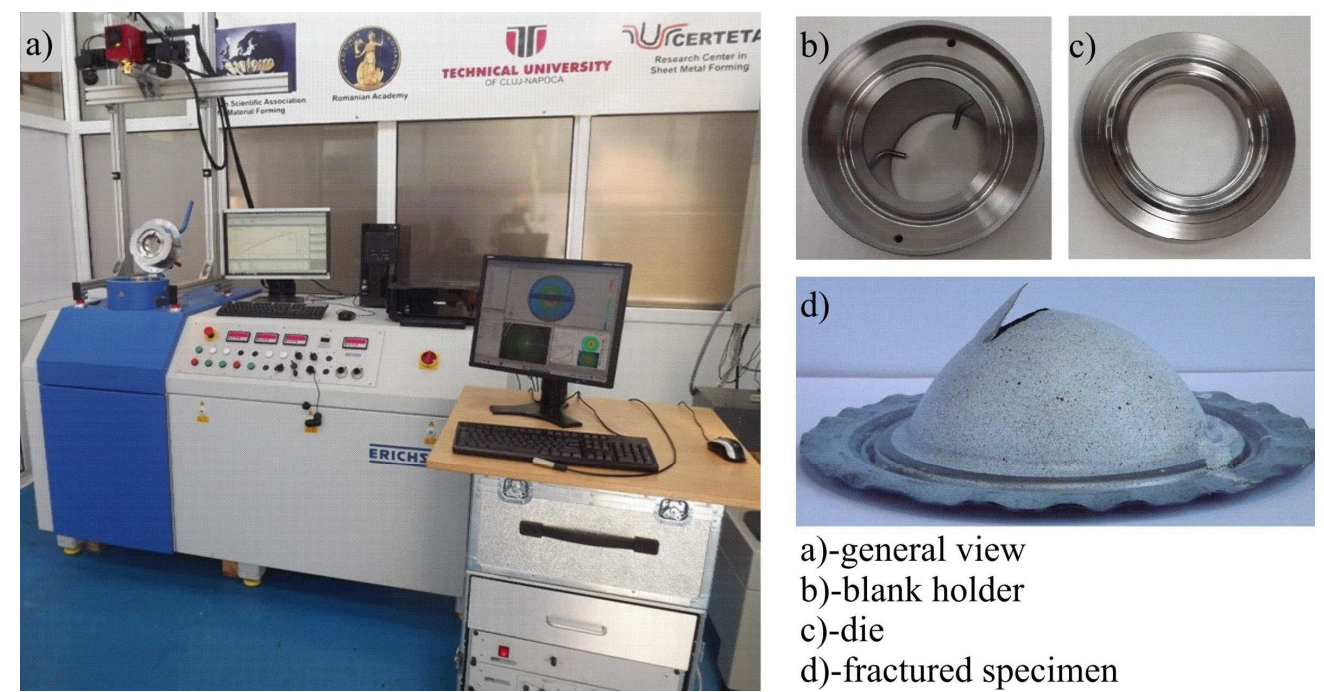

a)-general view

b)-blank holder

c)-die

d)-fractured specimen

Fig. 2. Experimental setup for the hydraulic bulge test

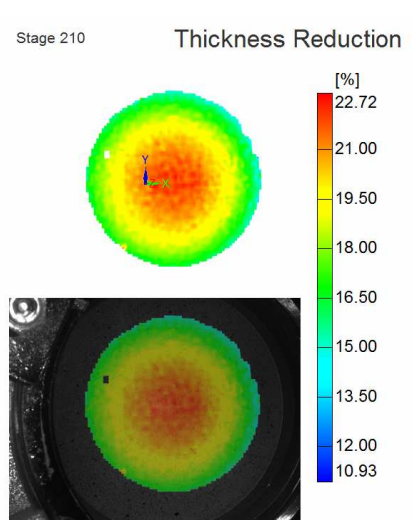

a)

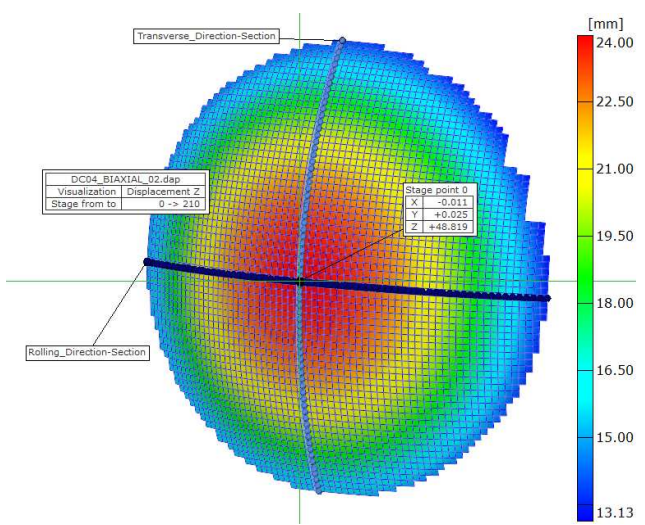

b)

Fig. 3. ARAMIS results: thickness reduction distribution (a) and the z-displacement distribution with the cross sections along the rolling direction (RD) and transverse direction (TD), respectively

(b) at a hydraulic pressure of $9.5 \mathrm{MPa}$ 


\section{AutoForm Sigma FEM simulation of hydraulic bulge test}

Finite Element model. In this paper a finite element simulation model of the hydraulic bulge test has been build using the commercially FE package AutoForm ${ }^{\text {plus }}$ R2 4.4 (Fig. 4a). The geometry and dimensions of the tools and sheet are the same as in the experiment. The model contains the tools (the die and the blank holder) and the blank. The eight parameters BBC 2005 material model has been used to model yield surface of the blank material whose input constitutive parameters are given in Table 1. The Swift approximation has been used to model the material hardening (see Fig. $1 \mathrm{~b}$ and Table 1).

The simulation process involves two steps: step 1 - closing the die, where the sheet is clamped between the die and blank holder; step 2 - the drawing, where the increasing pressure is applied on the bottom surface of the specimen. The Hydromech module with the active pressure option has been used to model the hydraulic bulge process, which allows setting a variable pressure during the process time, as shown in Fig. 4b. In order to minimize the effect of adaptive meshing, meshing parameters have been established as follows, initial element size: $3 \mathrm{~mm}$; radius penetration: 0.22 $\mathrm{mm}$; maximum element angle: $30^{\circ}$ and maximum displacement: $2.2 \mathrm{~mm}$.

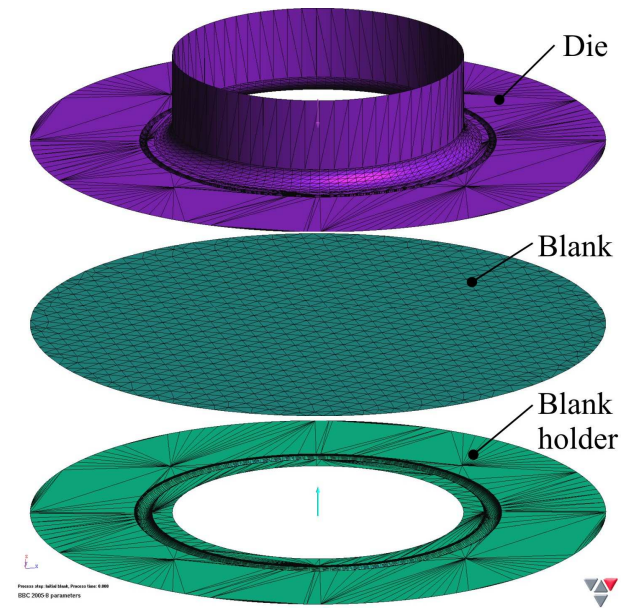

a)

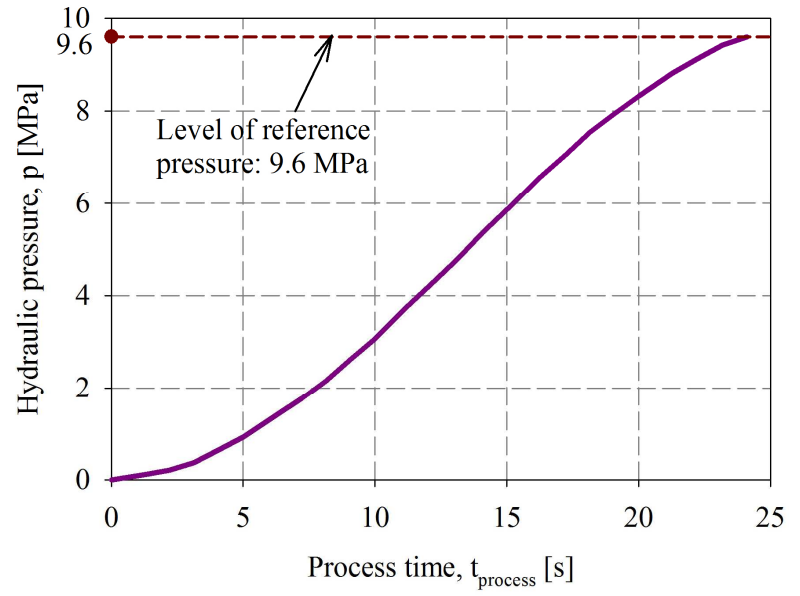

b)

Fig. 4. Finite element model of the bulge test (a) and the applied hydraulic pressure (b)

AutoForm Sigma-material noise analysis. AutoForm Sigma is a module in the FE simulation package AutoForm specialized for analyzing and improving the robustness of sheet metal products and processes. An AutoForm ${ }^{\text {plus }}$ Sigma R2 v4.4 noise analysis has been performed, where the noise variables are presented in Table 2.

Table 2 Assumed variation of mechanical property for the DC04 material

\begin{tabular}{lcccccc}
\hline \multicolumn{1}{c}{ Variables } & $\begin{array}{c}\text { Variable's name } \\
\text { in AutoForm Sigma }\end{array}$ & Current & Center & StdDev & Min & Max \\
\hline$\sigma_{45}[\mathrm{MPa}]$ & S_45 & 211 & 211 & 7.03 & 189.9 & 232.1 \\
\hline$\sigma_{90}[\mathrm{MPa}]$ & S_90 & 206 & 206 & 6.86 & 185.4 & 226.6 \\
\hline$\sigma_{\mathrm{b}}[\mathrm{MPa}]$ & S_b & 227 & 227 & 7.56 & 204.3 & 249.7 \\
\hline $\mathrm{r}_{0}[-]$ & r_0 & 1.96 & 1.96 & 0.130 & 1.568 & 2.352 \\
\hline $\mathrm{r}_{45}[-]$ & r_45 & 1.3 & 1.3 & 0.086 & 1.04 & 1.56 \\
\hline $\mathrm{r}_{90}[-]$ & r_90 & 2.19 & 2.19 & 0.146 & 1.752 & 2.628 \\
\hline
\end{tabular}

In this study, the authors consider only the variability of material property embedded in the eight parameters BBC 2005 yield criterion, used for the yield locus description. However, the yield stress along the rolling direction $\left(\sigma_{0}\right)$ has been eliminated from this study due to the software inability to include it as noise input. Also, the biaxial anisotropy coefficient $\left(\mathrm{r}_{\mathrm{b}}\right)$ has not been included in this study, because, as it was shown by Banabic $[13,14]$, its variability does not significantly influence 
the thickness, compared with the influence of biaxial stress $\left(\sigma_{b}\right)$. A variation of $\pm 10 \%$ and $\pm 20 \%$ respectively from the center value has been assumed for the yield stresses and anisotropy coefficients, respectively in Table 2, according to the recommendations in [15] for the mild steel. The standard deviation has been calculated as $\mathrm{StdDev}=(\operatorname{Max}-\mathrm{Min}) / 6$.

\section{Results and discussion}

Strain distribution. Fig. 5 shows the distribution of thickness strain versus distance from the bulged specimen pole provided by the finite element simulations in comparison with the experimental data. The thickness strain is considered in both the rolling direction (RD) and in the transverse direction (TD). From this diagram one may notice that the experimental curves are fitted sufficiently accurate by the simulation results, provided by the BBC 2005 yield model.

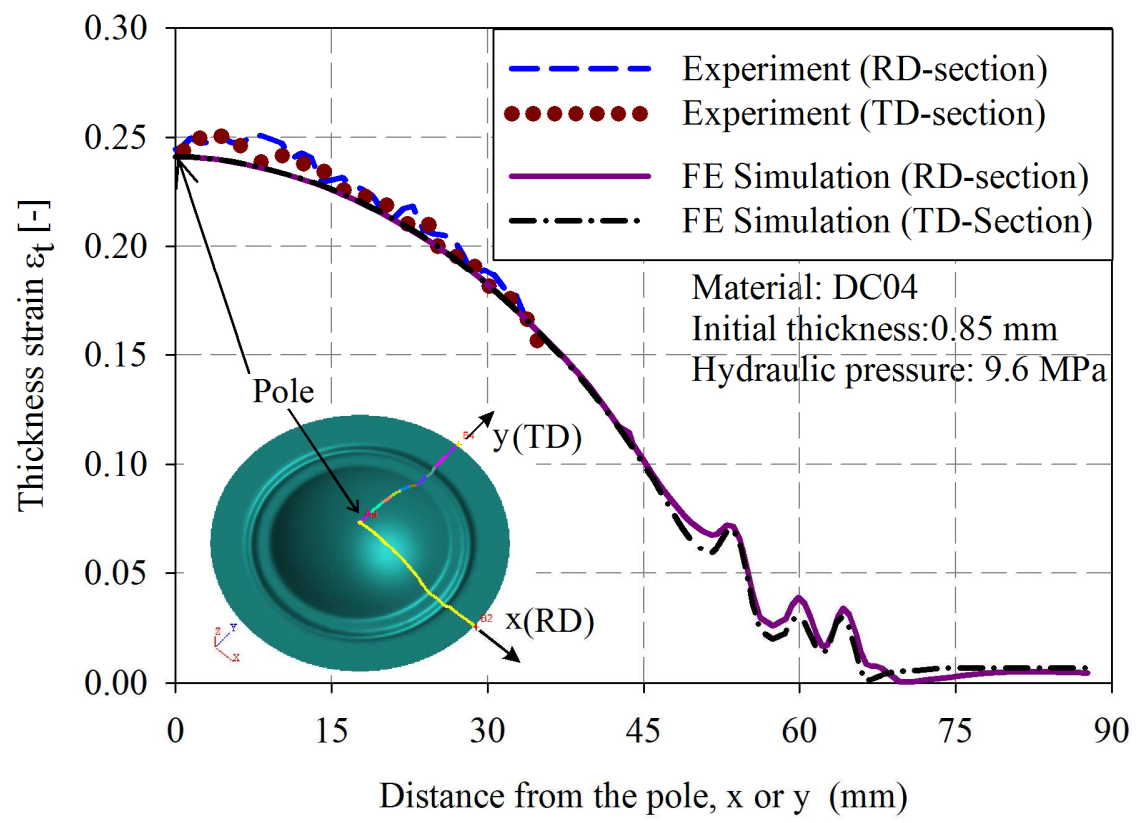

Fig. 5. Comparison between FE simulation and experiment for thickness-strain distribution

Influence analysis. The six material parameters in Table 2 were investigated, with 129 simulations performed and the variation of thickness response assessed as will present in the following.

In a sheet forming process, it is important to know which material parameters are dominant with respect to its influence on the thickness of formed part. Fig. 6 shows that at each zone on the specimen surface, a different material parameter is the dominant influence on thickness. Fig. 6 shows, as per expected, that the dominant variable in the polar zone is the biaxial stress. Four zones on the specimen surface (Fig. 6) were marked and labelled and will be further discussed in detail. Zone 1 is located in the polar zone and corresponds to a biaxial tensile stress states. Zones 2-4 are located near the fillet radius of the die and are characterized by a uniaxial tensile stress state. Additionally, the positions of these zones have been chosen at orientations of $0^{\circ}, 45^{\circ}$, and $90^{\circ}$ degrees, respectively with respect to the rolling direction (RD).

The Pareto charts and pie charts in Fig. 7 show the detailed influence of material parameters noise on the thickness at these zones of the specimen. The Pareto charts show the influence of material noise in a decreasing order from the left to the right. The influence can vary from 0 to 1 . A value of 0 means no influence so the result is absolutely independent on the parameter. A value of 1 means a strong influence so the result is completely dependent on the parameter. From Fig. 7a one may notice that the biaxial stress $\left(\sigma_{b}\right)$ has the greatest influence on the thickness in the polar zone (Zone $1)$. In Zone 2 , in the absence of yield stress $\left(\sigma_{0}\right)$, which has not been included in the study, the greatest effect on the thickness is assigned to the yield stress $\left(\sigma_{45}\right)$, followed by the yield stress 


\section{Innovation for Sustainable Development}

$\left(\sigma_{90}\right)$. In Zone 3 , at $45^{\circ}$ angle with the respect to the rolling direction, the dominant variable is the yield stress $\left(\sigma_{45}\right)$. In Zone 4 , placed at $90^{\circ}$ angle from the rolling direction the main effect is assigned to the yield stress $\left(\sigma_{90}\right)$, closely followed by the yield stress $\left(\sigma_{45}\right)$.

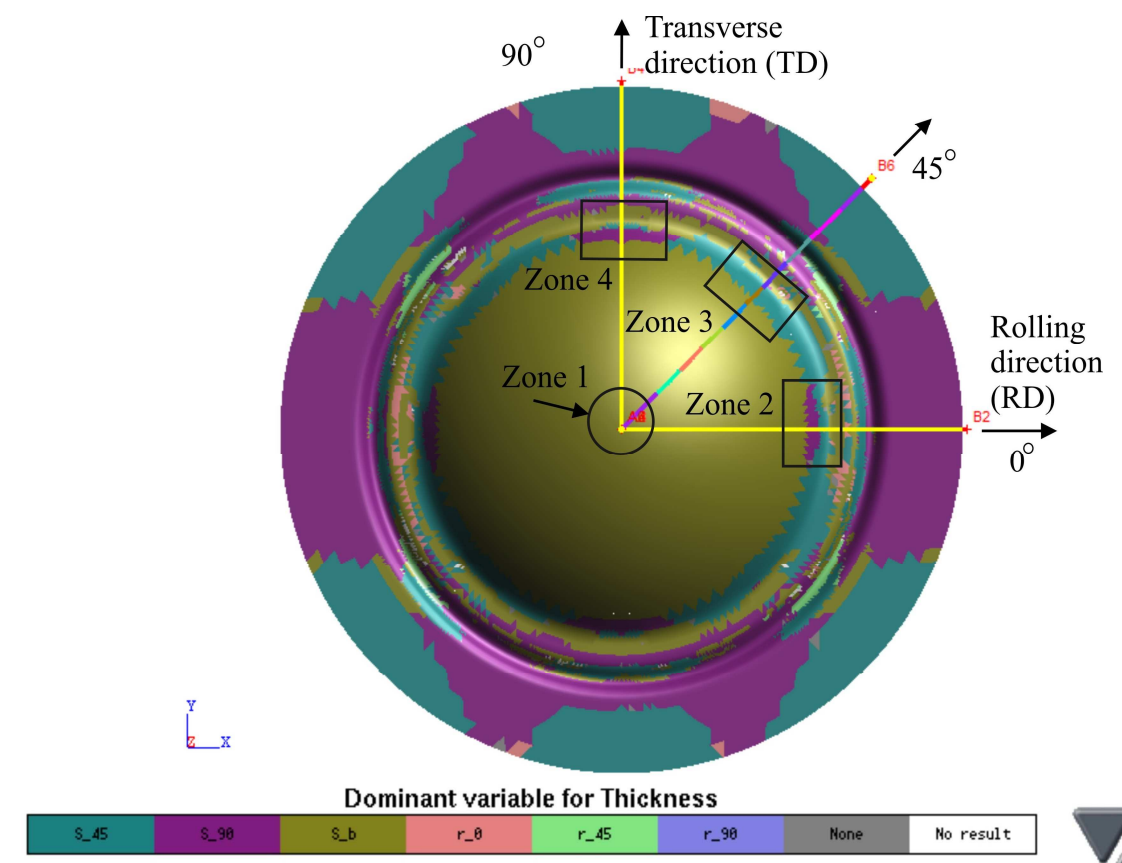

Process step: 20_drawing, Process time: 26.837

Fig. 6. The influence plot, showing the location within the part in which a different material parameter is the dominant design variable

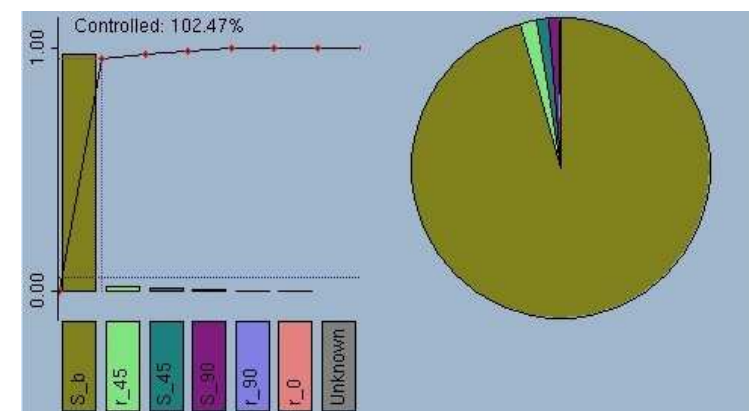

a) Zone 1

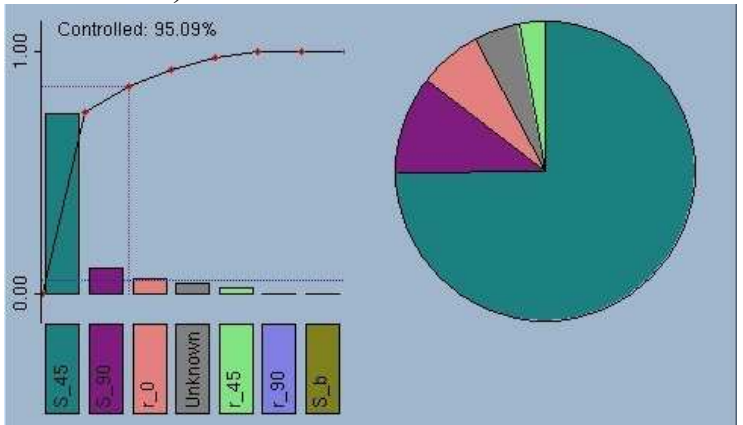

c) Zone 3

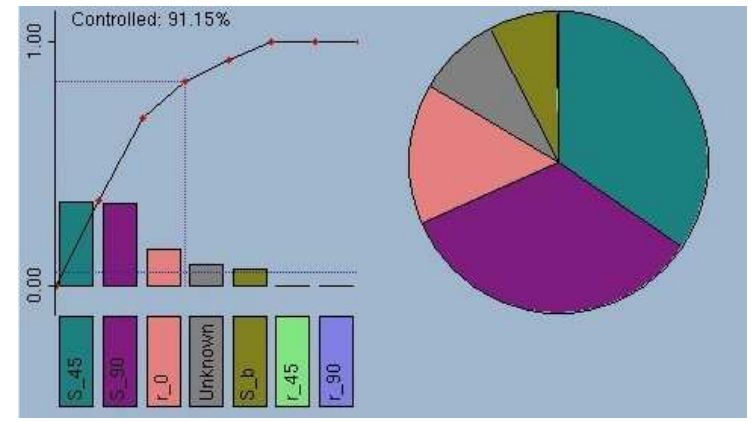

b) Zone 2
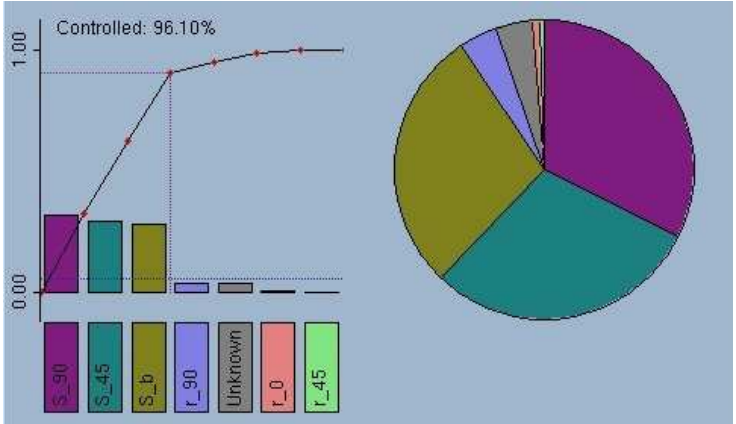

d) Zone 4

Fig. 7. AutoForm Sigma influence plots for the thickness response in the corresponding zones shown in Fig. 6

Sensitivity analysis. Fig. 8 shows the sensitivity of thickness in the considered zones. The read circles show that the sensitivity of thickness has positive sign. This means that the thickness increases in the corresponding zone when the corresponding material parameter is increased. The 
blue circle shows that the sensitivity of thickness has negative sign. This means that the thickness becomes smaller in the corresponding zone if the corresponding material parameter is increased. The yellow circles imply accidental results that are marked as untrusted. The green circles mean that the variation of material parameters does not change the thickness.

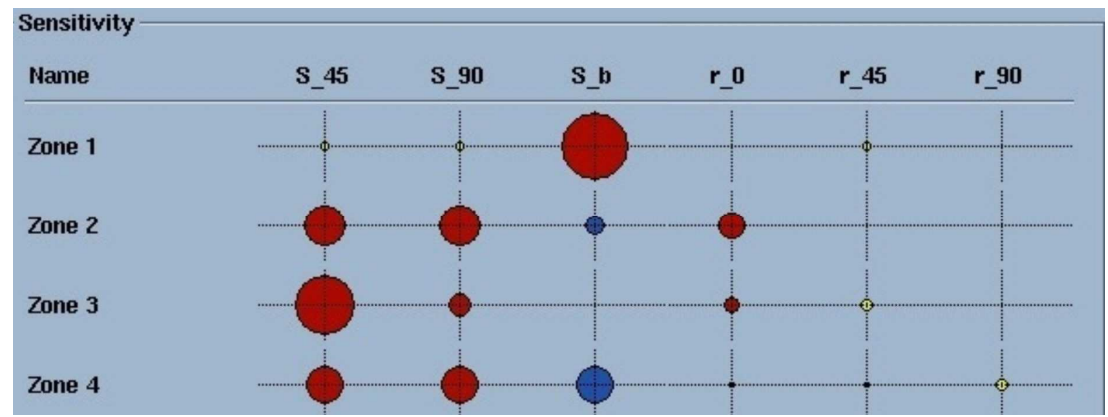

Fig. 8. Tab sensitivity in a global evaluation mode

The scatter plots in Fig. 9 shows the thickness variation and the selected material parameters in a xy scatter plot for 129 simulations carried out by AutoForm Sigma. The red circles denote the individual simulation results. The cross blue bar is the range of normal Inter Quantile Range (IQR) for both material parameters and thickness. The thick grey line indicates the correlation between the thickness and material parameter. From these charts one can see the variation of the thickness due to the variation of material property. For instance in Fig. 9a, the thickness increases from 0.58 to $0.71 \mathrm{~mm}$ when the biaxial stress increases form 206.4 to $245.7 \mathrm{MPa}$. From Fig. 9 one can see that thickness increases in all considered zone when the corresponding material parameter is increased, which already has been shown in Fig. 8.

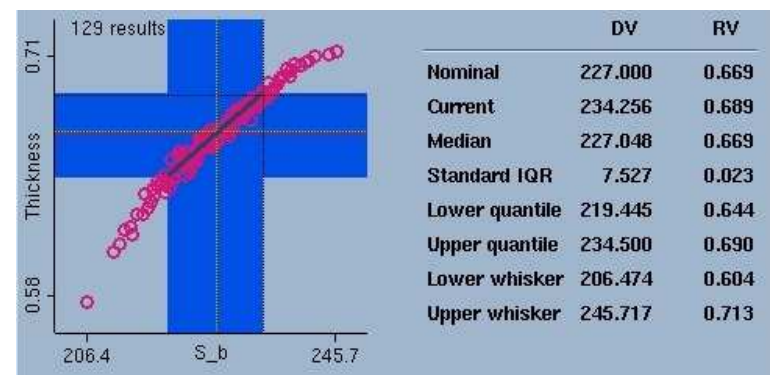

a) Zone 1

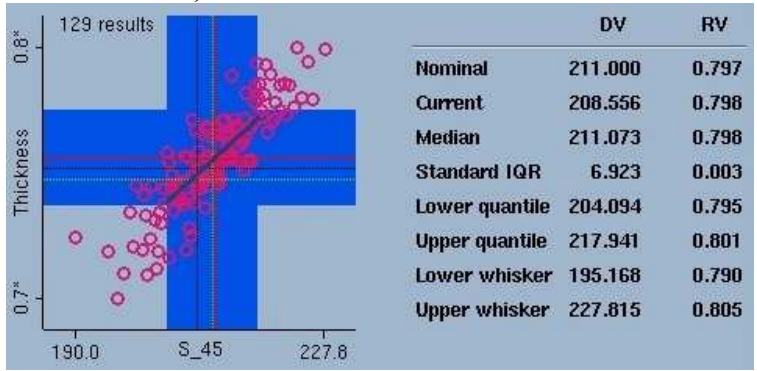

c) Zone 3

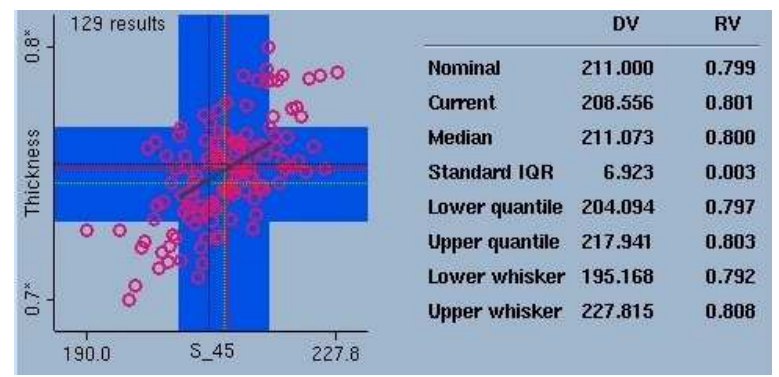

b) Zone 2

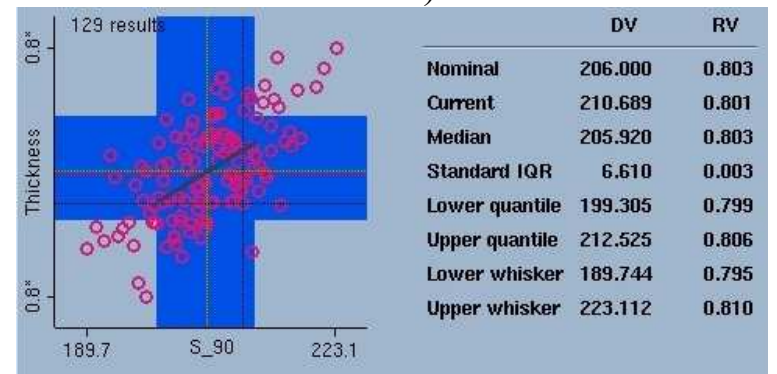

d) Zone 4

Fig. 9. Scatter plot of thickness and the corresponding material parameters in the considered zones shown in Fig. 6

\section{Conclusions}

In this paper the influence of material property variability on the thickness of sheet metal subjected to the hydraulic bulge test has been investigated using the AutoForm Sigma programme. Four zones within the specimen and six material parameters from the eight parameters BBC2005 yield criterion have been considered in the study. The obtained results show that in the zone characterized by a biaxial tensile stress state, the $\sigma_{\mathrm{b}}$ is the dominant influence on the thickness. In the zone 
characterized by a tensile stress state along the rolling direction, the dominant material parameter is $\sigma_{45}$. This may be explained by the fact that the yield stress $\sigma_{0}$ has not been considered in this study due to the inability of the AutoForm Sigma programme to include it as noise input. In the zone characterized by tensile stress state along the directions at $45^{\circ}$ and $90^{\circ}$ angle, respectively with respect to the rolling direction the dominant material parameter is $\sigma_{45}$ and $\sigma_{90}$, respectively.

From the three anisotropy coefficients $\left(r_{0}, r_{45}\right.$, and $\left.r_{90}\right)$ considered in this study only $r_{0}$ has influence in the zones characterized by tensile stress state along the directions at $45^{\circ}$ and $90^{\circ}$ angle measured from the rolling direction. As a consequence, in the hydraulic bulge test, the sheet thickness is most sensible to the variation of yield stresses than to the variation of anisotropy coefficients.

\section{Acknowledgements}

This paper was supported by the project POSDRU/89/1.5/S/52603, co-funded by the European Social Fund, by granting of postdoctoral fellowship and by the project PCCE 100/2010 by providing materials for the experiments.

\section{References}

[1] V. Karthik, R.J. Comstock, D.L. Hershberger, R.H. Wagoner, Variability of sheet formability and formability testing, J. Mat. Process Tech. 121 (2002) 350-362.

[2] L. Paraianu, I. Bichis, D. Banabic, Variability analysis of the mechanical parameters in order to determine the forming limit band, AIP Conf. Proc. 1353 (2011) 1511-1516.

[3] D. Banabic, M. Vos, Modelling of the forming limit band - a new method to increase the robustness in the simulation of sheet metal forming processes, Annals of CIRP. 56 (2007) 249-252.

[4] L. Paraianu, D.S. Comsa, D. Banabic, Sensitivity analysis of the mechanical parameters of the sheet metals influencing the forming limit curves, in: 3rd Int. Conf. Eng. Optim., Rio de Janeiro (2012).

[5] T. de Souza, B. Rolfe, Multivariate modelling of variability in sheet metal forming, J. Mat. Process. Tech. 203 Issues 1-3 (2008) 1-12.

[6] L. Marretta, R. Di Lorenzo, Influence of material properties variability on springback and thinning in sheet stamping processes: a stochastic analysis, Int. J. Adv. Manuf. Technol. 51 (2010) 117-134.

[7] P. Sugar, J. Sugarova, L. Morovič, P. Zemko, Analysis of dimensional accuracy of spun parts by Taguchi approach, Applied Mechanics and Materials. 217-219 (2012) 2423-2426.

[8] V. Gödel, C. Annen, M. Merklein, Material modelling for stochastic simulation, in: R. Kolleck (Ed.), Proc. 50 ${ }^{\text {th }}$ Conference of IDDRG 2010 Graz. Verlag der Technischen Universität Graz. (2010) 687-696.

[9] E. Atzema, M. Abspoel, P. Kömmelt, M. Lambriks, Towards robust simulations in sheet metal forming, International Journal of Material Forming. 2 (2009) 351-354.

[10] D. Banabic, Sheet Metal Forming Processes: Constitutive Modelling and Numerical Simulation, D. Banabic (Ed.), Springer, Berlin-Heidelberg, 2010.

[11] L. Lăzărescu, I. Nicodim, I. Ciobanu, D. S. Comşa, D. Banabic, Determination of material parameters of sheet metals using the hydraulic bulge test, Acta Metallurgica Slovaca, Vol. 19, No. 1, (2013) 4-12.

[12] ARAMIS V6.1.1-7 User Manual Software, GOM GmbH, Braunschweig (2009).

[13] D. Banabic, D. S. Comşa, M. Sester, M. Selig, W. Kubli, K. Mattiasson, M. Sigvant, Influence of constitutive equations on the accuracy of prediction in sheet metal forming simulation, in: P. Hora (Ed.), Numisheet 2008, Interlaken (2008) 37-42.

[14] D. Banabic, M. Sester, Influence of Material Models on the Accuracy of the Sheet Forming Simulation, Mater. Manuf. Process. 27 (2012) 304-308.

[15] AutoForm 4.4 Software Manual, AutoForm Engineering GmbH, Zurich (2010). 